\title{
Simulation and Experiment of Piezoelectric Injection Dispensing Valve
}

\author{
Guobin Lei ${ }^{1}$, Hong $\mathrm{Hu}^{1}{ }^{1}{ }^{*}$, Ruifeng $\mathrm{Li}^{2}$, Jian Chen ${ }^{1}$, and Junhao Shao ${ }^{1}$ \\ ${ }^{1}$ Harbin Institute of Technology Shenzhen Graduate School, Shenzhen 518055, China. \\ ${ }^{2}$ Inner Mongolia Power Machinery Research Institute, Hohhot, 010010, China \\ honghu@hit.edu.cn
}

Keywords: jetting dispensing, piezoelectric driven, flow field analysis, micro-displacement amplified.

\begin{abstract}
In recent years, dispensing systems have been used more widely, and various kinds of precise liquid distribution systems are in great demand for dispensing technology. In this paper, a jetting dispensing valve driven by piezoelectric ceramics is proposed, which takes advantage of the good response and output characteristics of piezoelectric ceramics. The jetting dispensing valve is mainly composed of a piezoelectric ceramic driver, micro-displacement amplifier, mechanical needle, and nozzle. The FLUENT is used to simulate the flow field analysis in the dispensing process. The phase diagram and velocity cloud diagram of the jetting process are obtained. Through the experiment, the influence law of feed pressure, dispensing cycle, and needle stroke on jetting dispensing is obtained. In the dispensing consistency experiment, in the case where the nozzle diameter is $0.1 \mathrm{~mm}$ and the nozzle and substrate distance is $4 \mathrm{~mm}$, a dispensing array having an average diameter of $0.311 \mathrm{~mm}$ is obtained, with a dispensing consistency error of $6 \%$. The efficiency and accuracy of the jetting dispensing valve have been improved.
\end{abstract}

\section{Introduction}

Fluid dispensing technology is the process of accurately dispensing glue in a controlled manner. With the development of technology, fluid dispensing technology has been widely used in various types of precision liquid distribution systems such as microelectronic packaging ${ }^{[1,2]}$, biomedicine ${ }^{[3]}$, microelectromechanical systems (MEMS) ${ }^{[4]}$, and additive manufacturing ${ }^{[5]}$.

Fluid dispensing technology can be divided into contact dispensing and non-contact dispensing according to the different movement modes of the nozzle. Such as time-pressure type dispensing, piston pump type dispensing and screw pump type dispensing are contact dispensing. This dispensing method must be in contact with the target surface during each dispensing process, and the dispensing needle needs to be lifted and lowered, so a high-precision displacement sensor is required. As a result, the dispensing efficiency is low, the speed is slow, and the consistency of the glue dots is poor. This dispensing technology has been gradually replaced by non-contact dispensing.

Non-contact dispensing is also called jet dispensing. The nozzle is kept at a certain distance from the target surface, and the glue near the nozzle outlet is forced to obtain a greater speed by an instantaneous high pressure, thereby separating from the nozzle, forming a droplet, and dripping at 
the target position to achieve dispensing. During the dispensing process, the needle has no displacement in the Z-axis direction. Compared with the contact dispensing technology, the non-contact dispensing greatly improves the dispensing speed, the dispensing efficiency is high, and the consistency of the glue points is good. There is no phenomenon of dispensing pollution, target surface damage caused by contact between the needle and the target surface in the contact dispensing. However, because the development time of non-contact dispensing technology is still short, the research on its injection mechanism is still immature, the glue point is relatively large, and the accuracy rate needs to be improved ${ }^{[6]}$.

Many universities and research institutions have conducted research on non-contact jet dispensing technology and achieved good results. Sun Hui ${ }^{[7]}$ used piezoelectric stack as the driving source, and used the diamond-shaped amplifying mechanism to amplify the displacement of the piezoelectric stack to achieve the stability and high efficiency of high-viscosity liquid dispensing. Jiao Xiaoyang et al. ${ }^{[8]}$ proposed a new piezoelectric-driven jet dispensing valve consisting of a piezoelectric stack, a lever-type micro-displacement amplifying mechanism, a valve stem, and a nozzle. They found that when the viscosity of the glue is $2 \mathrm{~Pa} \cdot \mathrm{s}$, the minimum diameter of the glue point obtained is $0.5 \mathrm{~mm}$, and the consistency error of the glue point is within $6 \%$. Nguyen et al ${ }^{[9]}$ proposed a new configuration of the high frequency jetting dispenser for integrated circuits (ICs) fabricating. The jetting dispenser can work well at $1000 \mathrm{~Hz}$ and the amplitude of needle motion can reach up to $0.3 \mathrm{~mm}$. Liu Yaxin et $\mathrm{al}^{[10]}$ combined the piezoelectric actuator with the needle type injection valve, and used the diamond-shaped amplifying mechanism to amplify the displacement of the piezoelectric actuator and then drive the needle to vibrate. The amplitude of the needle reached a range of $0.5 \mathrm{~mm}$, and the repeatability and accuracy of the distribution were controlled within $4 \%$ and $\pm 6 \%$.

\section{Structure and Principle}

The structure of the piezoelectric injection dispensing valve is shown in Fig. 1. It is mainly composed of a preloading fine adjustment system, piezoelectric ceramic driver, micro-displacement amplified flexible-hinge, mechanical needle, and nozzle.

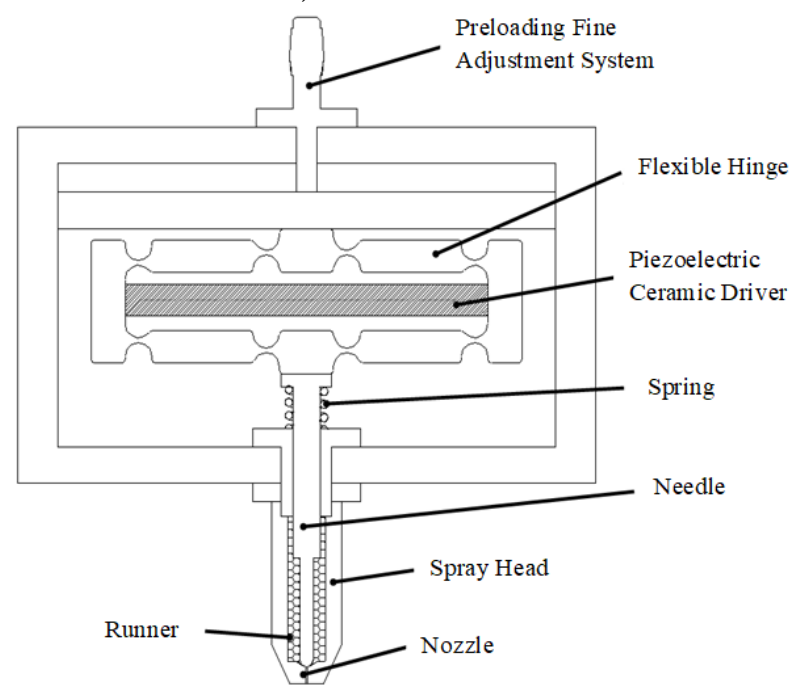

Fig. 1 Structure diagram of piezoelectric injection dispensing valve

The working principle of the piezoelectric injection dispensing valve is as follows:

1) In the state where the piezoelectric ceramic is not energized, the bottom end of the needle is attached to the upper end of the nozzle under the preloading fine adjustment system to prevent the liquid rubber from flowing out. 
2) After the piezoelectric ceramic is energized, the length is extended, and stretch the horizontal direction of the flexible hinge and compress it in the vertical direction, so that the needle moves upward under the action of the spring force; in this process, the liquid rubber is filled with the cavity of the needle and the nozzle under the action of the pressure.

3) The flexible hinge becomes longer in the vertical direction and restores to its original state after being powered off, and the needle moves rapidly downward under the action of the flexible hinge, the end spherical surface of the needle collides with the nozzle base, and the flowing liquid rubber is cut off. The liquid rubber under the spherical surface keeps the inertia ejected from the nozzle at a high speed to form the desired droplet on the target surface.

4) After the needle stays in the lowest position for a period of time, the piezoelectric ceramic is energized again, and the needle is stretched under the action of the spring force to start the next cycle of motion.

\section{Simulation}

\subsection{VOF Model}

In the fluid dynamics simulation problem studies in this paper, there are two phases of liquid and gas flow in the flow field. Both phases are continuous media. Therefore, the VOF model is used to simulate the interaction between the two phases of liquid and gas flow.

In the VOF model, different fluid components share a set of momentum equations, and by introducing a phase volume fraction the tracking of each computational unit interface is achieved ${ }^{[11]}$. The sum of the volume fractions of all phases is 1 in each control volume. Assuming that the volume fractions of the gas phase and the liquid phase are $\alpha_{1}$ and $\alpha_{2}$ respectively ${ }^{[11]}$, and no reaction occurs between the gas and liquid phases, the mixing density per unit volume can be expressed as:

$$
\rho=\alpha_{1} \rho_{1}+\alpha_{2} \rho_{2}
$$

Mass conservation equation:

$$
\frac{\partial \rho}{\partial t}+\frac{\partial(\rho u)}{\partial x}+\frac{\partial(\rho v)}{\partial y}+\frac{\partial(\rho w)}{\partial z}=0
$$

Where $\rho$ represents fluid density, and $u, v, w$ represents the velocity $\vec{v}$ components in the $x, y, z$ directions.

Let $q=1,2$, and bring equation (2) into (1), then the phase separation expression of the mass conservation equation is:

$$
\frac{\partial \alpha_{q}}{\partial t}+\vec{v} \cdot \nabla \alpha_{q}=0
$$

In the two-phase flow simulation process, the focus is on simulating phase interface tracking between gas and liquid. In the VOF model, the control volume method is used for interpolation to determine the position of the phase interface. There are two methods: geometric reconstruction method and material contact method. Fig. 2 is the diagram of interface reconstruction. The geometric reconstruction method in FLUENT is the most accurate. In this paper, the geometric reconstruction method is chosen to describe the gas-liquid phase interface. 


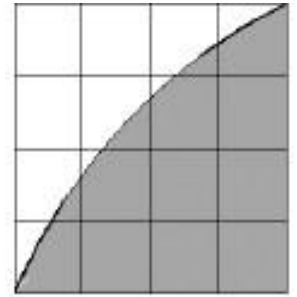

a) Actual interface

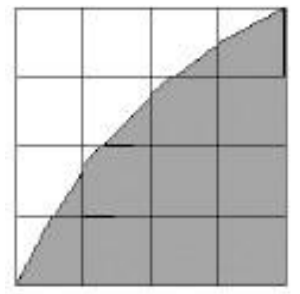

b) geometric reconstruction

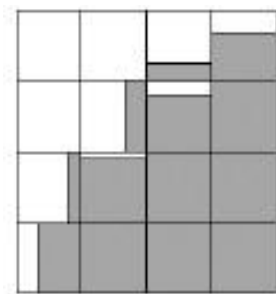

c) material contact

Fig. 2 Diagram of interface reconstruction

In the process of fluid injection, the influence of gravity and surface tension of the fluid needs to be considered, that is, the source term needs to be added in the mass conservation equation. Brackbill et al. ${ }^{[12]}$ proposed a continuous surface force model (CSF) in the VOF model. In the case where the surface tension is constant and only the normal force of the interface is considered, the relationship between the fluid surface pressure difference $\Delta p$ and the surface tension $\sigma$, the phase interface bidirectional curvature radius $R_{1}$ and $R_{2}$ can be expressed as:

$$
\Delta p=p_{2}-p_{1}=\sigma\left(\frac{1}{R_{1}}+\frac{1}{R_{2}}\right)
$$

Where $p_{1}, p_{2}$ represents pressure on both sides of the two fluid interfaces.

In FLUENT, when using the CSF formula, the surface curvature is calculated from the surface local gradient perpendicular to the interface. $n$ is the surface normal, then:

$$
n=\nabla \alpha_{q}
$$

The surface curvature $K$ is defined to distinguish the unit normal vector $\hat{n}$ :

$$
K=\nabla \cdot \hat{n}
$$

Where:

$$
\hat{n}=\frac{n}{|n|}
$$

The surface tension can be derived from changes in pressure across the surface. Surface force using divergence can be expressed as volume force. The expression is as follows:

$$
F_{v o l}=\sum_{\text {pairs }, i j, i<j} \sigma_{i j} \frac{\alpha_{i} \rho_{i} K_{j} \nabla \alpha_{j}+\alpha_{j} \rho_{j} K_{i} \nabla \alpha_{i}}{\frac{1}{2}\left(\rho_{i}+\rho_{j}\right)}
$$

If there are only two phases in a unit, then $K_{i}=-K_{j}, \nabla \alpha_{i}=\nabla \alpha_{j}$, so equation (8) can be simplified as:

$$
F_{\text {vol }}=\sigma_{i j} \frac{\rho K_{i} \nabla \alpha_{i}}{\frac{1}{2}\left(\rho_{i}+\rho_{j}\right)}
$$

Where $\rho$ represents the average density. Equation (9) shows that a unit surface tension source term is proportional to the unit average density.

\subsection{Simulation Results}

The fluid ejection process mainly includes the generation of droplets at the nozzle and the process of free movement in the air after droplet breakage. Therefore, the simplified model of the simulated object is the area of droplet formation at the nozzle, droplet breakage and free movement in the air. The calculation domain of the fluid ejection model is shown in Fig. 3. 


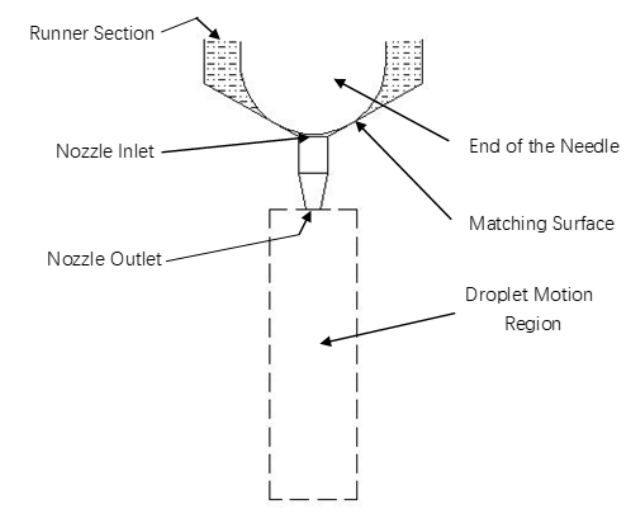

Fig. 3 Domain of Fluid injection simulation

The size associated with the nozzle: the nozzle diameter $d=0.1 \mathrm{~mm}$, and the length $l=0.5 \mathrm{~mm}$.

In the simulation analysis, an epoxide-resin glue with a viscosity of $1000 \mathrm{cps}$ and a surface tension coefficient of $325 \mathrm{dyn} / \mathrm{cm}$ is used as the research object. The glue injection process simulated in FLUENT is shown in Fig. 4.
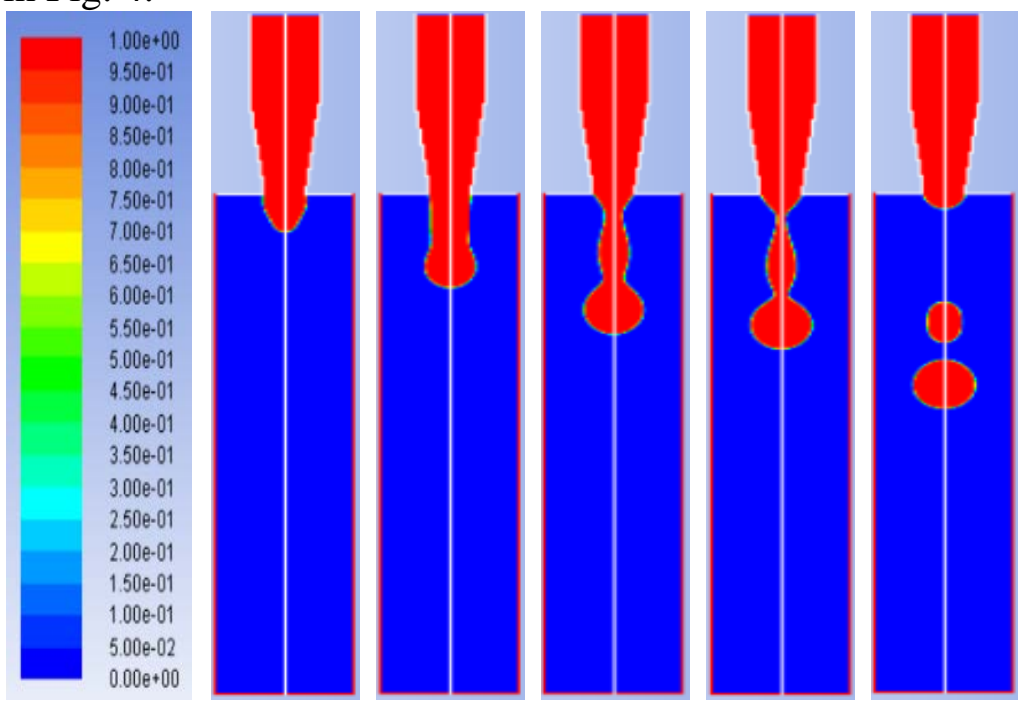

$0.2 \mathrm{~ms}$

$0.8 \mathrm{~ms}$

$1.4 \mathrm{~ms}$

$1.6 \mathrm{~ms}$

$2.4 \mathrm{~ms}$

a) Phase diagram of glue injection process

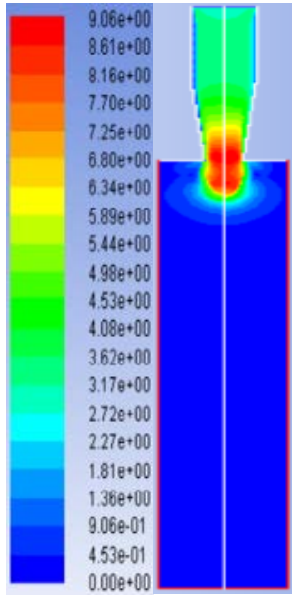

$0.2 \mathrm{~ms}$

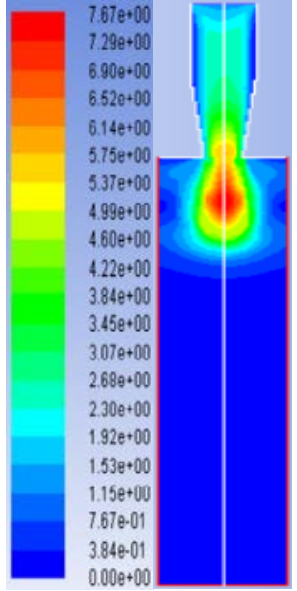

$0.8 \mathrm{~ms}$

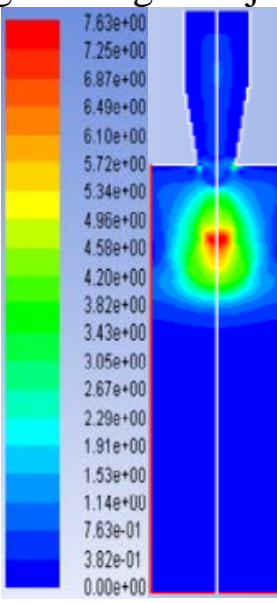

$1.4 \mathrm{~ms}$

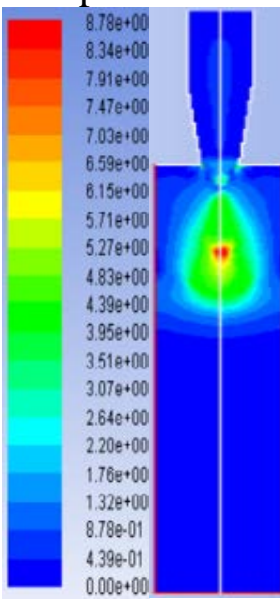

$1.6 \mathrm{~ms}$

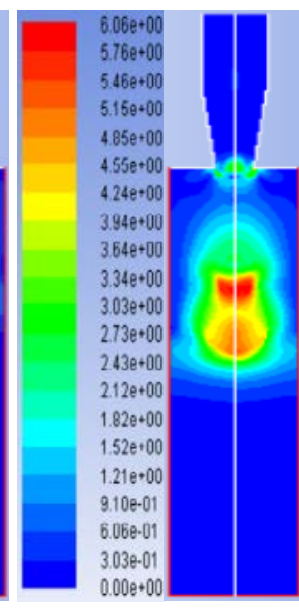

$2.4 \mathrm{~ms}$

b) Velocity cloud diagram of glue injection process

Fig. 4 Glue injection process and velocity cloud diagram 
From the figure, various stages of the droplet formation process can be observed: the generation stage, the elongation stage, the necking stage, and the fracture stage. When the needle is kept in the raised state, the glue is gradually filled into the cavity by the action of the supply air pressure and its own gravity, and even flows out from the nozzle. The needle then quickly falls and the fluid under the needle accelerates and is forced out of the nozzle. After the needle moves to the end, the nozzle is closed and the fluid stops flowing. It can be seen from the velocity cloud diagram that the velocity of the upper and lower parts of the fluid outside the nozzle is different, the fluid is elongated, the width of the tail is reduced, and the phenomenon of "necking" occurs. Under the action of the surface tension of the fluid, the neck is broken. The separated fluid is gradually rounded from the elongated shape to eventually form droplets.

\section{Experiment}

\subsection{Experimental Platform}

The experimental platform is mainly composed of a PC, a three-dimensional motion platform, a piezoelectric injection dispensing valve, a dispensing valve controller, and a constant pressure air supply system. In order to measure the weight and diameter of the glue points obtained in the experiment, a high-precision electronic scale with a measuring range of $50 \mathrm{~g}$ and a measuring accuracy of $0.1 \mathrm{mg}$, and an optical microscope, was used. The experimental platform is shown in Fig. 5.

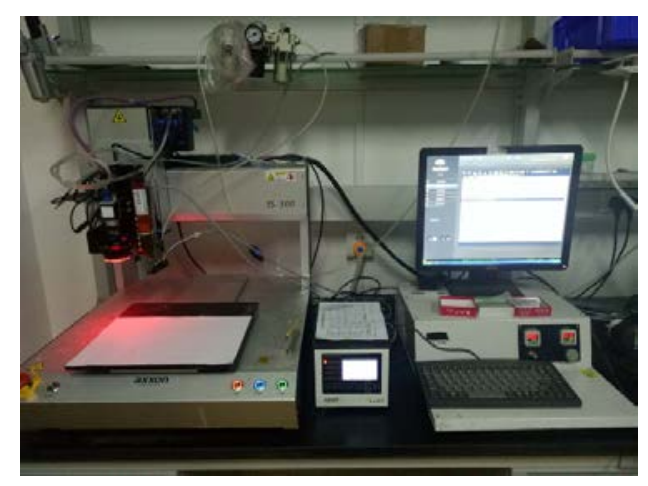

Fig. 5 The actual figure of experimental platform

\subsection{Effect of Feed Pressure on Droplet Volume}

In the experiment to test the relationship between feed pressure and dispensing quality, the dispensing process is set as follows: The viscosity of the glue is $1000 \mathrm{cps}$, the diameter of the nozzle is $0.1 \mathrm{~mm}$, the dispensing cycle is $4.0 \mathrm{~ms}$, the stroke of the needle is $70 \%$, and the distance between the nozzle and the substrate is $4 \mathrm{~mm}$. The feed pressure increased from 0 to $30 \mathrm{psi}$, with an interval of 5 psi. The experimental results of measuring the average mass and average diameter of the glue points are shown in Table 1 and Fig. 6. 
Table 1 Experimental results under different feed pressures

\begin{tabular}{ccccc}
\hline Feed pressure(psi) & Number & $\begin{array}{c}\text { Average } \\
\text { weight(mg) }\end{array}$ & $\begin{array}{c}\text { Average } \\
\text { diameter(mm) }\end{array}$ & $\begin{array}{c}\text { Standard } \\
\text { deviation(mm) }\end{array}$ \\
\hline 10 & 120 & 0.158 & 0.304 & 0.009 \\
15 & 120 & 0.178 & 0.313 & 0.012 \\
20 & 120 & 0.205 & 0.319 & 0.010 \\
25 & 120 & 0.233 & 0.327 & 0.013 \\
30 & 120 & 0.275 & 0.356 & 0.032 \\
\hline
\end{tabular}

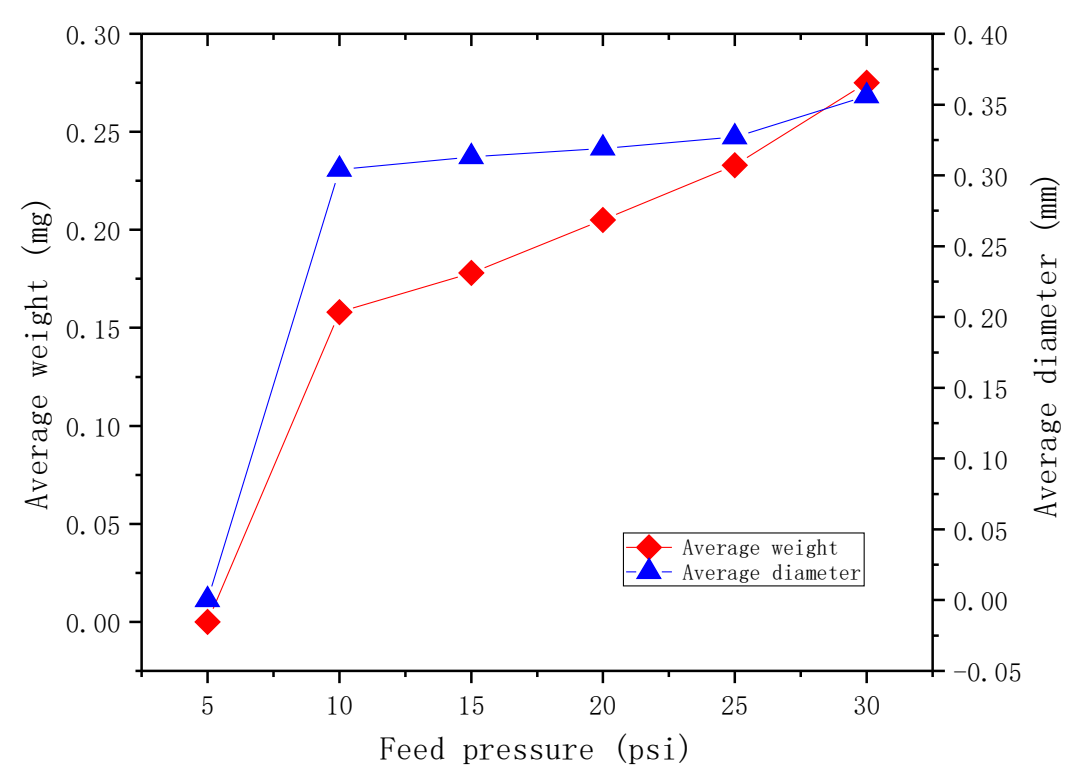

Fig. 6 Experimental results under different feed pressures

It can be seen that when the other parameters are constant, the injection condition is satisfied at a feed pressure of 5 psi, after that the droplet volume increases as the feed pressure increases.

\subsection{Effect of Dispensing Cycle on Droplet Volume}

In order to test the effect of dispensing cycle on the droplet volume, the viscosity is $1000 \mathrm{cps}$, the nozzle diameter is $0.1 \mathrm{~mm}$, the feed pressure is $15 \mathrm{psi}$, the stroke of the needle is $70 \%$, and the distance between the nozzle and the substrate is $4 \mathrm{~mm}$. The dispensing cycle is $3.0 \mathrm{~ms}, 3.5 \mathrm{~ms}, 4.0 \mathrm{~ms}, 4.5 \mathrm{~ms}$ and $5.0 \mathrm{~ms}$ respectively. The experimental results of measuring the average mass and average diameter of the glue points are shown in Table 2 and Fig. 7.

Table 2 Experimental results of different dispensing cycles

\begin{tabular}{ccccc}
\hline $\begin{array}{c}\text { Dispensing } \\
\text { cycle }(\mathrm{ms})\end{array}$ & Number & $\begin{array}{c}\text { Average } \\
\text { weight(mg) }\end{array}$ & $\begin{array}{c}\text { Average } \\
\text { diameter(mm) }\end{array}$ & $\begin{array}{c}\text { Standard } \\
\text { deviation(mm) }\end{array}$ \\
\hline 3.0 & 120 & 0.115 & 0.306 & 0.005 \\
3.5 & 120 & 0.127 & 0.309 & 0.011 \\
4.0 & 120 & 0.141 & 0.317 & 0.013 \\
4.5 & 120 & 0.187 & 0.332 & 0.016 \\
5.0 & 120 & 0.197 & 0.376 & 0.020 \\
\hline
\end{tabular}




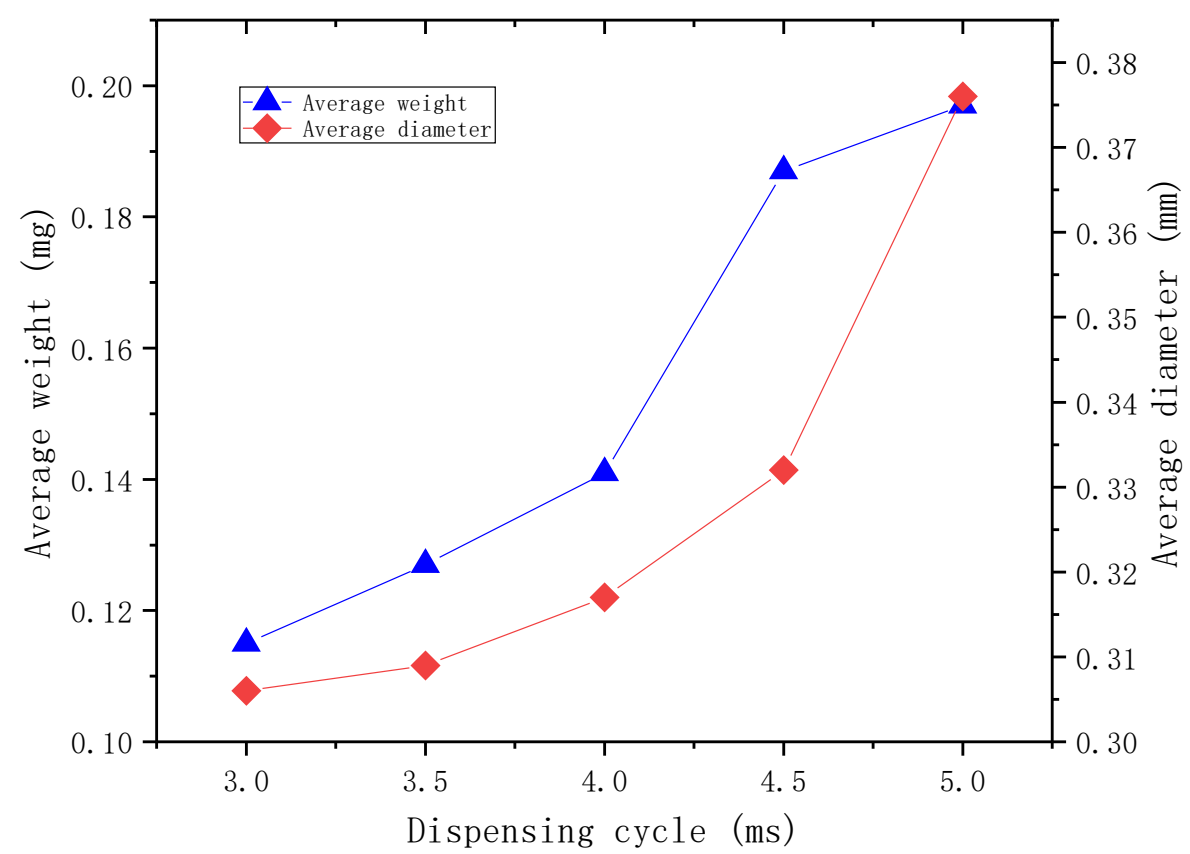

Fig. 7 Experimental results of different dispensing cycles

It can be seen that the droplet volume increases with the increase of the dispensing cycle while the other parameters are unchanged. When the dispensing cycle is less than $4 \mathrm{~ms}$, the dispensing cycle has little effect on the diameter of the droplet. After the dispensing cycle is greater than $4 \mathrm{~ms}$, the effect of the dispensing cycle on the diameter of the droplet is significantly increased.

\subsection{Effect of the Stroke of the Needle on the Droplet Volume}

In order to test the effect of the stroke of the needle on the droplet volume, the viscosity is $1000 \mathrm{cps}$, the nozzle diameter is $0.1 \mathrm{~mm}$, the feed pressure is $15 \mathrm{psi}$, and the dispensing cycle is $4.0 \mathrm{~ms}$. The stroke of the needle is $60 \%, 65 \%, 70 \%, 75 \%$, and $80 \%$, respectively. The experimental results of measuring the average mass and average diameter of the glue points are shown in Table 3 and Fig. 8.

Table 3 Experimental results for different needle strokes

\begin{tabular}{ccccc}
\hline $\begin{array}{c}\text { Needle } \\
\text { stroke }\end{array}$ & Number & $\begin{array}{c}\text { Average } \\
\text { weight(mg) }\end{array}$ & $\begin{array}{c}\text { Average } \\
\text { diameter(mm) }\end{array}$ & $\begin{array}{c}\text { Standard } \\
\text { deviation(mm) }\end{array}$ \\
\hline $60 \%$ & 120 & 0.128 & 0.294 & 0.007 \\
$65 \%$ & 120 & 0.150 & 0.304 & 0.009 \\
$70 \%$ & 120 & 0.178 & 0.312 & 0.011 \\
$75 \%$ & 120 & 0.252 & 0.349 & 0.018 \\
$80 \%$ & 120 & 0.285 & 0.381 & 0.029 \\
$85 \%$ & 120 & 0.308 & 0.394 & 0.033 \\
\hline
\end{tabular}




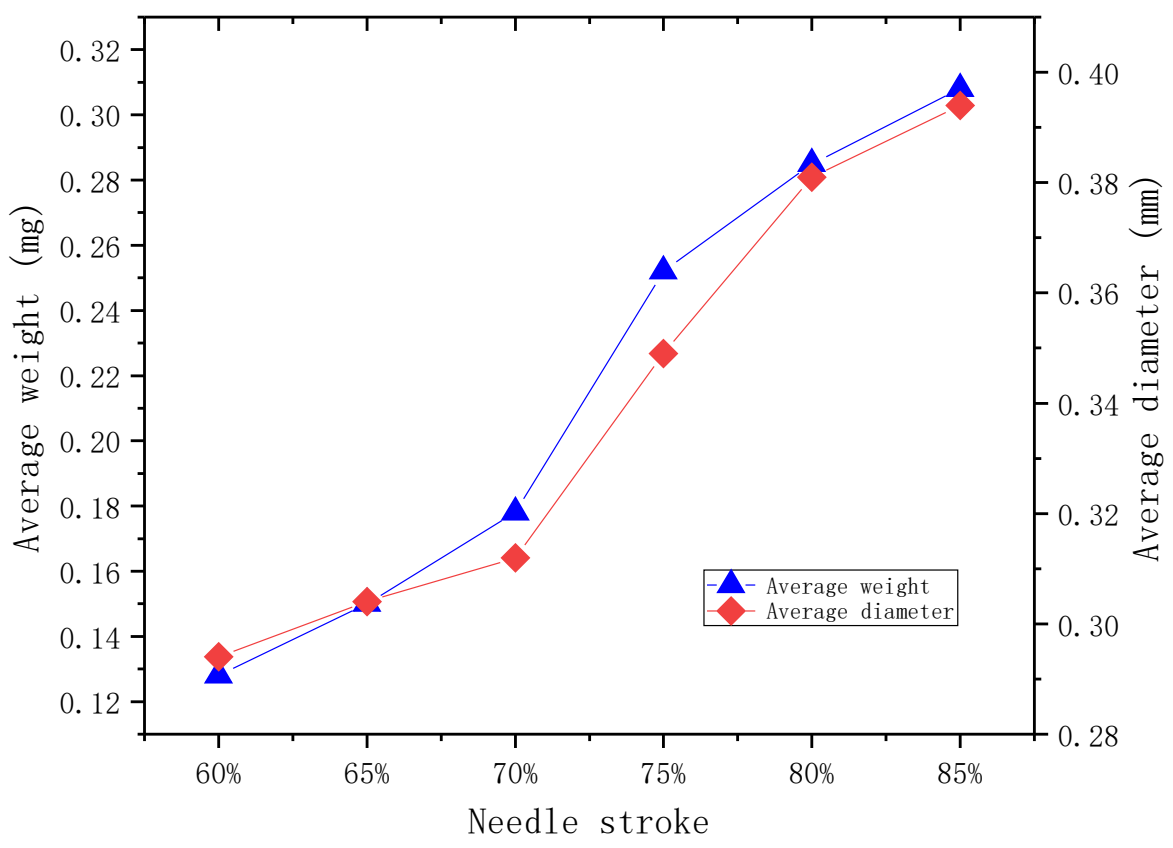

Fig. 8 Experimental results for different needle strokes

As can be seen from the graph, as the stroke of the needle increases, the droplet diameter and mass will also increase. And the effect of the stroke of the needle on the droplet volume is more significant. During the stroke of the needle from $60 \%$ to $85 \%$, the average diameter of the droplets increased by more than $30 \%$.

\subsection{Experiment of Dispensing Consistency}

It is intuitive to observe the dispensing consistency of the dispensing valve by dispensing the graphic array under the same dispensing conditions. The experimental conditions: the viscosity is $1000 \mathrm{cps}$, the nozzle diameter is $0.1 \mathrm{~mm}$, the dispensing cycle is $4.0 \mathrm{~ms}$, the stroke of the needle is $70 \%$, the feed pressure is 15psi, and the distance between the nozzle and the substrate is $4 \mathrm{~mm}$. The experimental results are shown in Fig. 9. The diameter of the glue points is measured by an optical microscope, the average diameter is $\Phi 0.311 \mathrm{~mm}$, the maximum diameter is $\Phi 0.332 \mathrm{~mm}$, the minimum diameter is $\Phi 0.291 \mathrm{~mm}$, and the upper and lower deviation was $6 \%$. The dispensing consistency is good. 


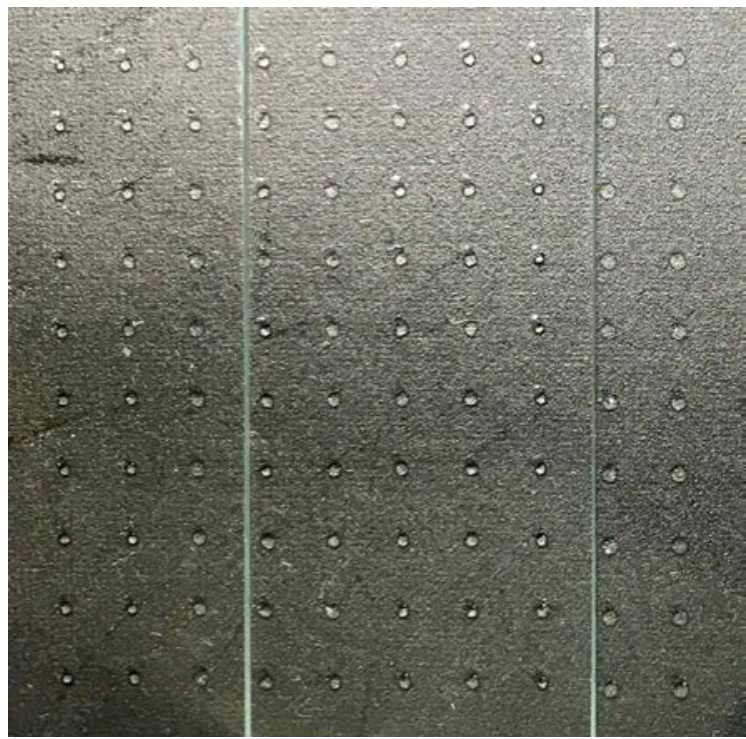

a) The $10 \times 10$ dispensing array

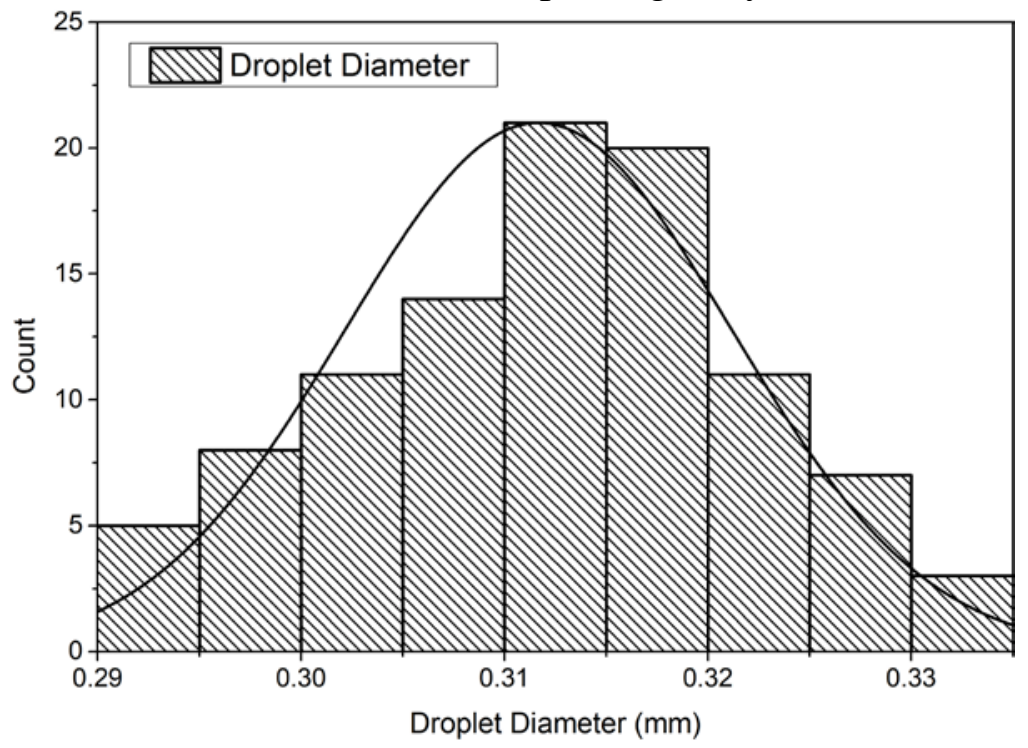

b) The normal distribution of droplet diameter

Fig. 9 Dispensing array and statistical results

\section{Summary}

Drop-on-demand micro-injection technology has broad application prospects. In this paper, for the demand of non-contact micro dispensing valves in industries such as electronic packaging, a jetting dispensing valve driven by piezoelectric ceramics is proposed. Theoretical and simulation analysis is carried out on droplet ejection. The experimental research on the parameters affecting the injection process of the device is carried out, and the effect of feed pressure, dispensing cycle and needle stroke on the injection dispensing is obtained. In the dispensing consistency experiment, in the case where the nozzle diameter is $0.1 \mathrm{~mm}$ and the nozzle and substrate distance is $4 \mathrm{~mm}$, a dispensing array having an average diameter of $0.311 \mathrm{~mm}$ is obtained, the upper and lower deviations are only $6 \%$, and the dispensing consistency is excellent, and the dispensing valve dispensing cycle takes only a few milliseconds, which provides a more efficient and accurate dispensing method for industries such as electronic packaging. 


\section{Acknowledgment}

The authors gratefully acknowledge the support provided by the Shenzhen Government Fund JSGG20170412143346791 and JCY20170413105740689.

\section{References}

[1] Sun Daoheng, Gao Junchuan, Du Jiang. Advances in fluid dispensing technology for micro-electronics packaging[J]. China Mechanical Engineering, 2011, 22(20): 2513-2519.

[2] Zhao Yixiang, Chen Xindu, Chen Xin. An overview of fluid dispensing technology for micro-electronics packaging[J]. Chinese Hydraulics \& Pneumatics, 2006(2): 52-54.

[3] Landers R, Pfister A, Hubner U, et a1. Fabrication of soft tissue engineering scaffolds by means of rapid prototyping techniques[J]. Journal of Material Science, 2002, 37(15): 3107-3116.

[4] Zhang Lue, Hu Hong, Cao Yong, et al. A non-contact dispensing inker for dice marking of wafer detection. IEEE Transactions on Components, Packaging and Manufacturing Technology, 2011, 1(9): 1486-1493.

[5] Nguyen Q H, Han Y M, Choi S B, et a1. Dynamic characteristics of a new jetting dispenser driven by piezostack actuator[J]. IEEE Transaction on Electronics Packaging Manufacturing, 2008, 31(3): 248-259.

[6] Li J P, Deng G L. Technology development and basic theory study of fluid dispense--A review[C]//Proceedings of the Sixth IEEE CPMT Conference on High Density Microsystem Design and Packaging and Component Failure. 2004: 198-205.

[7] Sun hui. Research on non-contact dispensing technology for micro-volume of high viscosity adhesives [D]. Harbin: Harbin Institute of Technology, 2011.

[8] Jiao Xiaoyang, Liu Jianfang, Gu Fengchun. Analysis and research on jetting ability of the jet dispensing valve driven by a piezostack [J]. Journal of Sichuan University (Engineering Science Edition), 2013, 45(02): 193-198.

[9] Nguyen Q H. Optimal design of a jetting dispenser actuated by a dual piezoactuator[J]. Proceedings of SPIE-The International Society for Optical Engineering, 2014, 9057(15): 4177-4180.

[10] Liu Yaxin, Zhao Yatao, Lu Shizhou. Research on needle-type micro jet dispensing systems driven by piezoeletric actuator for high viscous liquids[J]. Journal of Xi'an Jiaotong University, 2015, 49(06): 90-95.

[11] Wu Kailiang. Jetting type of precision dispensing valve design and analysis[D]. Harbin Institute of Technology, 2012.

[12] Brackbill J U, Kothe D B, Zemach C. A continum method for modeling surface tension[J]. Journal of Computational Physics, 1992, 100(2): 335-354. 